\title{
Pharmacodynamics of siRNARANKL and Oestrogen Loaded MSNs-CADY on Human Periodontal Ligament Stem Cells with Porphyromonas gingivalis infection
}

\section{Liang Song ( $\nabla$ slenll@163.com )}

Fifth People's Hospital of Shanghai Fudan University https://orcid.org/0000-0001-5068-4859

\section{Xiaojun Shi}

Fifth People's Hospital of Shanghai Fudan University

\section{Fengling Hu}

Fifth People's Hospital of Shanghai Fudan University

\section{Huijuan Chen}

Fifth People's Hospital of Shanghai Fudan University

\section{Bin Xu}

Fifth People's Hospital of Shanghai Fudan University

\section{Yinghua Li}

Fifth People's Hospital of Shanghai Fudan University

Yan Li

Central South University Third Xiangya Hospital

\section{Research article}

Keywords: Periodontitis, PDLSCs, RANKL, Oestrogen, MSNs

Posted Date: January 6th, 2020

DOI: https://doi.org/10.21203/rs.2.20120/v1

License: (a) (i) This work is licensed under a Creative Commons Attribution 4.0 International License. Read Full License 


\section{Pharmacodynamics of siRNA $A_{\text {RANKL }}$ and Oestrogen Loaded MSNs-CADY on Human Periodontal Ligament Stem Cells with Porphyromonas gingivalis infection}

Shi Xiaojun, ${ }^{1} \mathrm{Hu}$ Fengling, ${ }^{1}$ Chen Huijuan,,${ }^{1} \mathrm{Xu}$ Bin,,${ }^{1} \mathrm{Li}$ Yinghua,${ }^{2} \mathrm{Li}$ Yan, ${ }^{3 *}$ and Liang Song ${ }^{1 *}$

Shi Xiaojun_shixiaojun@5thhospital.com

Hu Fengling hufengling1077@163.com

Chen Huijuan 289881928@qq.com

Xu Bin_ xbwander@sina.com

Li Yinghua zhulihan@zju.edu.cn

Li Yan*_1y.zxt@163.com

Liang Song* slenll@163.com

1 Department of Stomatology, The Fifth People's Hospital of Shanghai, Fudan

University, 801 Heqing Road, Shanghai 200240, China

2 Central Laboratory, The Fifth People's Hospital of Shanghai, Fudan

University, 801 Heqing Road, Shanghai 200240, China

3 Department of Health Management, The Third Xiangya Hospital, Central South University, Tongzipo Road 138, Changsha, 410013, Hunan Province, China.

Shi Xiaojun and $\mathrm{Hu}$ Fengling are co-first author, they contributed equally to this work.

Correspondence should be addressed to Liang Song; slenll@163.com and Li Yan; ly.zxt@163.com. 


\section{Abstract}

Background: Periodontitis irreversibly invades and destroys periodontal supporting tissues, loses the ability of periodontal regeneration and restoration, and eventually leads to tooth loosening and loss. periodontal ligament stem cells (PDLSCs) hold great promises for periodontal tissue regeneration which was the potential target of periodontitis treatment, siRNA $A_{R A N K L}$ and oestrogen can help PDLSCs maintain normal function, however, it was very difficult for siRNA $\mathrm{RANKL}_{\text {and }}$ aestrogen to get into PDLSCs. Here, Cell penetrating peptide CADY was modified on the surface of siRNARANKL and oestrogen loaded mesoporous silica nanoparticles (MSNs) to carry them into Porphyromonas gingivalis infected PDLSCs, Then further affect the proliferation of PDLSCs.

Methods: 120-150 $\mathrm{nm}$ Mesoporous silica nanoparticles (MSNs) was prepared, and the biocompatibility, loading capacity and drug release properity were tested; MSNs was modified by penetrating peptide CADY and the prepared MSNs/CADY was loaded with siRNARANKL and oestrogen; In vitro drug release of siRNARANKL/MSNs-CADY and oestrogen/MSNs-CADY was tested by using semi-permeable dialysis bag diffusion; Cellular uptake and internalization of FITC-Labeled MSNs and FITC-Labeled MSNs-CADY was observed by use of Laser confocal microscopy; Finally, the effect of siRNARANKL and oestrogen loaded MSNs-CADY on cell proliferation of Porphyromonas gingivalis infected 
human periodontal ligament stem cells was tested by MTT assay.

Results: according to the results, MSNs-CADY with a concentration of $6.25-200 \mathrm{ug} / \mathrm{mL}$ have no toxic to PDLSCs; $24.6 \mathrm{mg}$ oestrogen and 0.5 mM siRNA RANKL $_{\text {can }}$ be loaded into $1 \mathrm{mg}$ of MSNs-CADY; and drug loaded MSNs-CADY nanodrug carriers can release siRNA $A_{\text {ANKL }}$ and oestrogen stably for at least $48 \mathrm{~h}$; After modification with cell penetrating peptide CADY, more MSNs-CADY can be taken by PDLSCs.

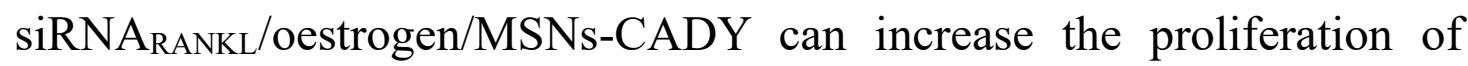
PDLSCs significantly.

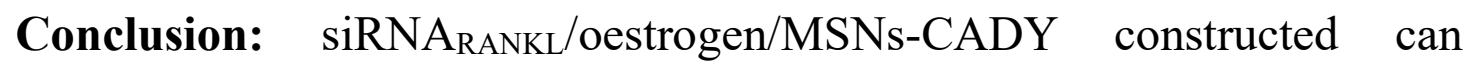
significantly improve the cell proliferation of P-gingivalis infected PDLSCs, this nano drug carrier has the potential to be used in PDLSCs -based periodontitis treatment, this work provided a useful theoretical basis and therapeutic ideas for the treatment of periodontitis.

\section{Keywords}

Periodontitis, PDLSCs, RANKL, Oestrogen, MSNs,

\section{Background}

Periodontitis is a chronic inflammatory disease of periodontal supporting tissues. It mainly relies on local immune response. It irreversibly invades and destroys periodontal supporting tissues, loses the ability of 
periodontal regeneration and restoration, and eventually leads to tooth loosening and loss, it is an inflammatory disease negatively affecting up to $15 \%$ of adults worldwide [1]. Plaque bacteria and their metabolites are the initiating factors of periodontal disease. Porphyromonas gingivalis (P.gingivalis) is one of the most virulent periodontal pathogens. It is closely related to the severity and recurrence of periodontal lesions [2]. It is generally agreed that P.gingivalis infection effect bone formation and bone resorption and then effect the reconstruction of the periodontal ligament (PDL) [3]. Recent studies reported that periodontal ligament stem cells (PDLSCs) hold great promises for periodontal tissue regeneration, PDLSCs possess the capacity for self-renewal and multipotent differentiation, and are able to form cementum/PDL-like tissue in vivo $[4,5]$.

As we know, the Receptor activator of NF- $\mathrm{kB}$ ligand/osteoprotegerin (RANKL/OPG) system plays a very important role in the regulation of osteoclastogenesis in bone systems [6], Lipopolysaccharide (LPS) is the most important pathogenic factor of P.gingivalis, the main pathogen of periodontitis. It can promote the up-regulation of lymphocyte inflammatory factor secretion, activate osteoclast expression, and lead to alveolar bone destruction and absorption [7]. It has been reported that LPS of P.gingivalis can up-regulate the expression of RANKL in periodontal ligament cells and osteoblasts [8]. Oestrogen (E2) is a 
hormone that affecting biological processes that include bone formation, angiogenesis and autoimmunity [9-11]. It was also reported that exogenous E2 enhanced the proliferation and osteogenic differentiation of hPDLSCs during in vitro culture [12]. Theoretically, the expression of RANKL should be decreased in hPDLSCs which infected by P.gingivalis, and exogenous E2 at an appropriate concentration can also help hPDLSCs maintain normal function. Therefore, drug carrier with high drug loading capacity and good release performance was needed.

The main purpose of this study was to construct a mesoporous nano drug carrier which can improve the internalization efficiency of siRNARANKL and oestrogen, and then to evaluate the pharmacodynamics of the SiRNARANKL and oestrogen loaded drug carrier on proliferation of P-gingivalis infected PDLSCs.our study provided a useful theoretical basis and therapeutic ideas for the treatment of periodontitis.

\section{Methods}

\section{Construction of CADY modified MSNs}

Firstly, Mesoporous silica nanoparticles with an diameter of about 120 $\mathrm{nm}$ were prepared according to Liu's method with some modification [13]. In brief, $5 \mathrm{~mL} \mathrm{NH}_{3} \cdot \mathrm{H}_{2} \mathrm{O}, 5 \mathrm{~mL} \mathrm{ddH}_{2} \mathrm{O}$ and $50 \mathrm{~mL}$ ethanol were mixed in and flasked, then the mixed solution was heated to $60{ }^{\circ} \mathrm{C}$. and then $2 \mathrm{~mL}$ tetraethoxysilane (TEOS) was added drop wise at a speed of $0.5 \mathrm{~mL} / \mathrm{min}$ after diluted in ethanol. Subsequently, the solution was heated at $60{ }^{\circ} \mathrm{C}$ 
under continuous stirring for $4 \mathrm{~h}$. finally, then unmodified mesoporous silica nanoparticles from the reaction was collected by centrifugation at $12000 \mathrm{~g}$ and washed 3 times with ethanol, and dried at $60{ }^{\circ} \mathrm{C}$ overnight. Secondly, $1 \mathrm{~mL}$ of the carboxy-modified (3-aminopropyl) triethoxysilane (APTES) reacted with $3 \mathrm{~mL}$ of succinic anhydride $(1 \mathrm{mg} / \mathrm{mL})$ in the presence of dichloromethane at room temperature for $24 \mathrm{~h}$, then $5 \mathrm{~mL}$ carboxy-modified APTES was added to $10 \mathrm{mg}$ MSNs in ethanol and stirring for $24 \mathrm{~h}$. Thereafter, $10 \mathrm{mg}$ MSNs-COOH were separated and washed with water for several times. FITC-APTES was prepared by added $200 \mathrm{uL}$ FITC $(1 \mathrm{mg} / \mathrm{mL})$ into $5 \mathrm{~mL}$ APTES in the presence of methanol under dark and dry condition. Then the $1 \mathrm{~mL}$ FITC-APTES was added to $10 \mathrm{mg}$ MSNs-COOH and stirring for $24 \mathrm{~h}$. Finally, $100 \mathrm{mg}$ of methoxy polyethylene glycol succinimidyl carbonate (mPEG-SC) was dissolved in $25 \mathrm{~mL}$ of dimethyl sulfoxide (DMSO), and $20 \mathrm{~mL}$ of this DMSO solution was added to the $10 \mathrm{mg}$ FITC-labeled MSNs, followed by stirring at $4{ }^{\circ} \mathrm{C}$ for $24 \mathrm{~h}$.

Thirdly, CADY peptide (GLWWKAWWKAWWKSLWWRKRKRKA) was purchased from Sangon Biotech Co. (Shang hai; HPLC-purified; purity $>95 \%$, identified by MS). Cross-linking reagents EDC and NHS was used to covalently conjugated CADY was onto MSNs, $3 \mathrm{mg}$ of MSNs-COOH was dissolved in $1 \mathrm{ml}$ of MES buffer ( $\mathrm{pH}$ 6.0), then $6 \mathrm{mg}$ of EDC and $6.4 \mathrm{mg}$ of NHS was added and the mixture was stirred at 
$4{ }^{\circ} \mathrm{C}$ for $6 \mathrm{~h}$. Then the solution was washed for times with additional $\mathrm{ddH}_{2} \mathrm{O}$. Then $100 \mu \mathrm{l}$ of CADY solution $(10 \mathrm{mg} / \mathrm{ml})$ was addedand the mixture was stirred for another 4 hours at $4{ }^{\circ} \mathrm{C}$, washed three times in PBS.

\section{siRNA $A_{\text {RANKL }}$ and oestrogen Loaded}

About $15 \mathrm{mg}$ of MSNs-CADY was dispersed into the solution with $\operatorname{siRNA} A_{\text {RANKL }}(50 \mu \mathrm{g} / \mathrm{mL})$ and oestrogen $(1 \mathrm{mg} / \mathrm{mL})$ under at $4{ }^{\circ} \mathrm{C}$ for $48 \mathrm{~h}$.

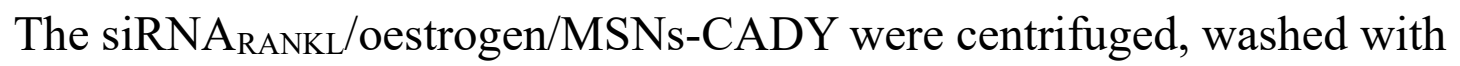
PBS for three times, and then dried under vacuum at room temperature overnight.

In Vitro Drug Release of siRNA $A_{\text {RANKL }} / M_{\text {MNNS-CADY and }}$ oestrogen/MSNs-CADY

In vitro release of siRNARANKL/MSNs-CADY and oestrogen/MSNs-CADY was evaluated by using semi-permeable dialysis bag diffusion. $10 \mathrm{mg}$ of siRNARANKL/MSNs-CADY and oestrogen/MSNs-CADY were dispersed in $5 \mathrm{~mL}$ of PBS (pH 7.4) separately with stirring and ultrasonication. Then, release mediums were placed in semipermeable dialysis bags and immersed into $15 \mathrm{~mL}$, respectively, at $37{ }^{\circ} \mathrm{C}$ with gentle shaking $(120 \mathrm{rpm})$. At different time intervals, $4 \mathrm{~mL}$ of the PBS from semipermeable dialysis bags was withdrawn to measure the amount of released oestrogen by human estradiol (E2) ELISA kit purchased form mibio (mlbio, Hangzhou, china, 
www.mlbio.cn), and the release of siRNARANKL was test by use of nanodrop (ThermoFish). An equal amount (4 mL) of fresh medium was added to the semipermeable dialysis bags after taking a measurement.

\section{PDLSCs Cell culture}

PDLSCs cells were purchased from Saibaokang (Shanghai) Biotechnology Co., Ltd. (http://www.icellbioscience.com, shanghai, china) and cultured in the media wih a-MEM (Gibco BRL, Gaithersburg, MD, USA) supplemented with 10\% FBS, 2mM L-glutamine (Invitrogen, Carlsbad, CA, USA), $100 \mathrm{U} / \mathrm{ml}$ penicillin, and $100 \mathrm{mg} / \mathrm{ml}$ streptomycin (Gibco BRL) at $37{ }^{\circ} \mathrm{C}$ in a humidified atmosphere of $5 \% \mathrm{CO}_{2}$.

\section{Cellular Internalization}

The PDLSCs cells were seeded on $60 \mathrm{~mm}$ dishes (Corning Incorporated). $100 \mathrm{mg} / \mathrm{mL}$ FITC-labeled MSNs and FITC-labeled MSNs-CADY was added to the cells, cells were washed with PBS four times after incubated for $12 \mathrm{~h}$, then stained with Lysotracker Red DND-99 (Beyotime Institute of Biotechnology, Haimen, China) for 30 min, washed with PBS 3 times, added $2 \mathrm{~mL}$ of $4 \%$ paraformaldehyde, then stained with $10 \mu \mathrm{g} / \mathrm{mL}$ DAPI (2-(4-amidinophenyl)-6-indolecarbamidine dihydrochloride, Sigma), with PBS three times. Cells were observed and imaging was taken with a laser scanning confocal microscope (Leica TCS sp5, Germany).

\section{In Vitro cell viability test}

The effect of of MSNs-CADY and siRNARANKL/oestrogen/MSNs-CADY 
on the viability of PDLSCs was tested by using the 3-[4, 5-dimethylthylthiazol-2-yl]-2,5 diphenyltetrazolium broide (MTT) assay. The PDLSCs were seeded in a 96-well plate at a density of 5000 cells per well. Thereafter, MSNs-CADY with different concentrations $(200,100$, $50,25,12.5$ and $6.25 \mathrm{mg} / \mathrm{mL}$ ) were added to the medium, and then the cells were incubated for 24 and $48 \mathrm{~h}$. Finally, the cell viability was assessed using the MTT assay.

Establishment of a model of Porphyromonas gingivalis infected PDLSCs

P.gingivalis was purchased from The Global Bioresource Center (ATCC, www.atcc.org), The strain was inoculated in fresh agar medium containing $5 \%$ sterile defibrized sheep blood, $1 \%$ hemin chloride and $0.1 \%$ vitamin $\mathrm{K}$. Anaerobic culture at $37{ }^{\circ} \mathrm{C}$ for $5-7$ days, monoclonal colonies were culated in liquid medium for $24 \mathrm{~h}$, centrifuged for $10 \mathrm{~min}$ at $6000 \mathrm{~g}$ and $4{ }^{\circ} \mathrm{C}$ to collect bacteria. The bacteria were washed once by PBS and suspended in antibiotic-free cell culture medium. Then add 150 $\mu \mathrm{L}$ of $1 \times 10^{9}$ P.gingivalis into well cultured PDLSCs, all the test should be completed in 48 hours.

\section{Characterization}

The morphology and structure of nanoparticles were characterized by a field-emission scanning electron microscope (S-4800) at accelerating voltages of 5 and $200 \mathrm{kV}$. 
Zeta potential measurements: Electrokinetic measurements were obtained at $25{ }^{\circ} \mathrm{C}$ between each functionalization step using a ZetaSizer Nano apparatus (Malvern Instruments, UK), in a PBS buffer solution (25 mM) at $\mathrm{pH}$ 7.2. The zeta potential was calculated using the Schmolukowski model.

\section{Statistical Analysis}

One-way ANOVA was used for statistical analysis of data to confirm the presence of significant differences in measured parameters between test groups; Individual points were compared using a Student's t test, and differences were considered significant at $p<0.05$. All the experimental data were expressed as mean $\pm \mathrm{SD}$. All the experiments were repeated at least three times $(n=3)$.

\section{Results}

\section{Preparation and Characterization of MSNs, MSNs-COOH,}

\section{MSNs-CADY}

The MSNs with the diameter about $120 \mathrm{~nm}$ were first synthesized, then the black MSNs were modified with carboxyl, finally, cell penetrating peptide CADY was attached on the MSNs by use of EDC and NHS reaction. TEM images of each nanoparticle were shown in figure 1, and zeta potential of each nanoparticle indicated that the CADY has been modified on MSNs and $120 \mathrm{~nm}$ of MSNs-CADY was prepared successfully. 


\section{Cell Compatibility of MSNs-CADY}

PDLSCs is relatively fragile, the cell compatibility of nano-drug carrier is very important. MTT cell activity detection kit was used to detect the effect of different concentrations of nanoparticles on cell viability, as shown in figure 2, MSNs-CADY has high PDLSCs compatibility, PDLSCs of cell viability was $94.5 \%$ and $88.4 \%$ even treated with 200 $\mathrm{ug} / \mathrm{mL}$ MSNs-CADY for $24 \mathrm{~h}$ and $48 \mathrm{~h}$.

Cellular Uptake and Internalization of FITC-Labeled MSNs and FITC-Labeled MSNs-CADY

Suit nano-drug carriers should effectively carry drugs into cells, membrane-penetrating peptides such as CADY has been reported can improve the cellular internalization efficiency of drug-carriers [14-16].

Here, we constructed MSNs-CADY and then FITC was labeled on the surface of MSNs-CADY, $100 \mu \mathrm{g} / \mathrm{ml}$ of FITC-labeled MSNs and FITC-labeled MSNs-CADY were separately incubated with PDLSCs for $12 \mathrm{~h}$. As shown in figure 3, the FITC relative light density of FITC-Labeled MSNs was $13.4 \%$ and the FITC-Labeled MSNs-CADY26.7\%, the result indicated that CADY modification can significantly improve the efficiency of PDLSCs cellular uptake and internalization of nano drug carrier.

In Vitro Drug Releasing Property of siRNARankL/MSNs-CADY and oestrogen/MSNs-CADY 
Mesoporous silica nanoparticles can be used as drug carriers because of their good sustained release properties, here, the in vitro drug releasing property of siRNARANKL/MSNs-CADY and oestrogen/MSNs-CADY was tested, as shown in figure 4, MSNs-CADY has good releasing property to siRNA $_{\text {RANKL }}$ and oestrogen both in siRNARANKL/MSNs-CADY and oestrogen/MSNs-CADY. After $10 \mathrm{~h}$ of rapid release, siRNARANKL and oestrogen loaded in MSNs-CADY can be released stably and slowly for at least $48 \mathrm{~h}$. In addition, the MSNs-CADY prepared here has high drug loading capacity, $1 \mathrm{mg}$ of MSNs-CADY can load $24.6 \mathrm{mg}$ oestrogen and $0.5 \mathrm{mM}$ SiRNARANKL.

\section{Effect of siRNA RANKL $_{\text {oestrogen/MSNs-CADY on the cell }}$ proliferation of P-gingivalis infected PDLSCs}

The direct effect of bacterial infection will cause to greatly reduction of cell proliferation. Here, we tested the effect of nano-drug carriers on the proliferation of p-gingivalis infected PDLSCs, as the result shown in figure 5, after $48 \mathrm{~h}$ culture, the number of PDLSCs in control group, infected PDLSCs and infected PDLSCs+drug carrier group was about $445.7 \times 104,117.9 \times 104$ and $390.5 \times 104$ separately, the results showed

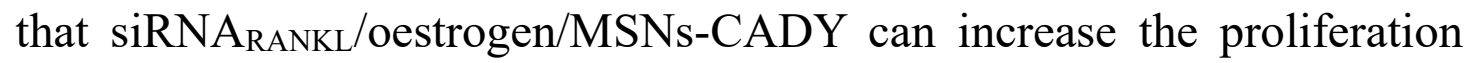
of PDLSCs significantly.

\section{Discussion}

Periodontitis is a common complex disease with relatively late onset, 
which is characterized by inflammatory destruction of periodontal attachment and bone loss. PDLCs) are able to participate in connective tissue remodeling while suffering mechanical stress from orthodontic appliances. If the disease is not treated in time, repeated attacks over a long period of time can cause dental defects, and then affect oral function. In clinic, systemic or oral local therapy is often used $[2,17,18]$. The P-gingivalis is the main pathogen of periodontal disease [19]. It was reported that damage to periodontal ligament stem cells (PDLSCs) during illness is one of the reasons why the disease is difficult to eradicate [20, 21]. PDLSCs represent a unique mesenchymal stem cells (MSC) population as demonstrated by their capacity to generate a cementum/periodontal ligament - like tissue in vivo, highlighting their available and reliable features for periodontal regeneration. Previous research data showed that PDLSCs akin to the immunomodulatory properties exhibited by other stromal populations [4, 22], PDLSCs has been studied as the target of periodontitis treatment. Decreasing the expression of RANKL by using siRNARANKL in P-gingivalis infected PDLSCs is helpful for cells to maintain normal physiological functions [8]. Oestrogen (E2) also reported can enhanced the proliferation and osteogenic differentiation of hPDLSCs during in vitro culture. Both the

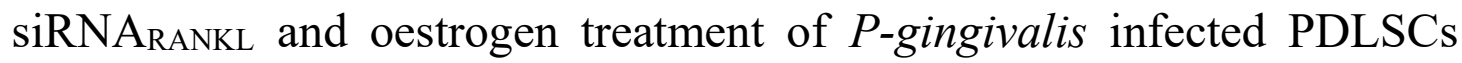
need a drug-carrier which with good PDLSCs biocapacity, high drug 
loading capacity and good drug release property, and the drug carrier also should has high cell internalization efficiency.

In this paper, MSNs-CADY was constructed to use as such nano-carrier, according the results, MSNs-CADY prepared here having all the features required above, firstly, it has high cell compatibility, secondly, based on the mesoporous structure of mesoporous silica nanoparticles, the drug carrier has high siRNA $A_{\text {RANKL }}$ and oestrogen loading capacity, and good drug release properity. After modification with cell penetrating peptide CADY, more MSNs-CADY can be taken by PDLSCs. In 2017, Lin et al reported that E2 was able to enhance the proliferation of human periodontal ligament stem cells [23], And in 2016, Jens Gruber et al deliveried siRNA with polyoma JC virus-like particles can decrease the expression of RANKL in rats significantly [24]. Here, we studied the effect of siRNARANKL and oestrogen loaded MSNs-CADY on the cell proliferation of P-gingivalis infected PDLSCs, and results shown that siRNARANKL/oestrogen/MSNs-CADY can increase the proliferation of $P$-gingivalis infected PDLSCs significantly.

\section{Conclusion}

In short, siRNARANKL/oestrogen/MSNs-CADY we constructed here can significantly improve the cell proliferation of $P$-gingivalis infected PDLSCs, this nano drug carrier has the potential to be used in PDLSCs -based periodontitis treatment, this work provided a useful theoretical 
basis and therapeutic ideas for the treatment of periodontitis. In addition, we will continue to evaluate the efficacy of the nano drug carrier in periodontitis treatment in vivo in the future and to provide preclinical theoretical basis for the treatment of periodontitis.

\begin{abstract}
Abbreviations
PDLCs: Periodontal ligament cells; PDLSCs: Periodontal ligament stem cells; MSC: Mesenchymal stem cells; MSNs: Mesoporous silica nanoparticles; P.gingivalis: Porphyromonas gingivalis; PDL: Periodontal ligament; RANKL/OPG: Receptor activator of NF-кB ligand/osteoprotegerin; LPS: Lipopolysaccharide; E2: Oestrogen; TEOS:

Tetraethoxysilane; APTES: 3-aminopropyl) triethoxysilaneMethoxy; mPEG-SC: polyethylene glycol succinimidyl carbonate; DMSO: Dimethyl sulfoxide; DAPI: (2-(4-amidinophenyl)-6-indolecarbamidine dihydrochloride; MTT: 3-(4,5-dimethyl-2-thiazolyl)-2,5-diphenyl-2-H-tetrazolium bromide; ANOVA: Analysis of variance.
\end{abstract}

\title{
Acknowledge
}

This work was supported by Shanghai Municipal Planning Commission of Science and Research Fund (Grant no. 201740230), Talent Development Plan funded by the Fifth People's Hospital of Shanghai, Fudan University (no. 2017WYRCJY02).

\section{Funding}

This work was supported by Shanghai Municipal Planning Commission of Science and Research Fund (Grant no. 201740230), Talent Development Plan funded by the Fifth People's Hospital of Shanghai, Fudan University (no. 2017WYRCJY02).

\section{Availability of data and materials}

All the data generated or analysed during this study are included in this published article. 


\section{Authors' contributions}

Li Yan, and Liang Song was the head of this subject. Shi Xiaojun performed the design and management of experimental scheme and completed the preparation, testing and characterization of nanoparticles, Hu Fengling completed the culture of PDLCs. Chen Huijuan completed the imaging work by use of Laser confocal microscopy. Xu Bin completed the in vitro drug release of nanoparticle testing. Li Yinghua completed the MTT assay. All authors read and approved the final manuscript.

\section{Ethics approval and consent to participate}

Not applicable.

\section{Consent for publication}

Not applicable.

\section{Competing interests}

The authors declare that they have no competing interests

\section{Reference}

1. Albandar JM: Epidemiology and risk factors of periodontal diseases. Dent Clin North Am 2005, 49(3):517-532, v-vi.

2. Lin L, Li C, Liu J, Zhang D, Zhao J, Kou Y, Yu N, Pan Y: Virulence genes of Porphyromonas gingivalis W83 in chronic periodontitis. Acta Odontol Scand 2009, 67(5):258-264.

3. Golz L, Memmert S, Rath-Deschner B, Jager A, Appel T, Baumgarten G, Gotz W, Frede S: LPS from P. gingivalis and hypoxia increases oxidative stress in periodontal ligament fibroblasts and contributes to periodontitis. Mediators Inflamm 2014, 2014:986264.

4. Seo BM, Miura M, Gronthos S, Bartold PM, Batouli S, Brahim J, Young M, 
Robey PG, Wang CY, Shi S: Investigation of multipotent postnatal stem cells from human periodontal ligament. Lancet 2004, 364(9429):149-155.

5. Cha Y, Jeon M, Lee HS, Kim S, Kim SO, Lee JH, Song JS: Effects of In Vitro Osteogenic Induction on In Vivo Tissue Regeneration by Dental Pulp and Periodontal Ligament Stem Cells. J Endod 2015, 41(9):1462-1468.

6. Silva I, Branco JC: Rank/Rankl/opg: literature review. Acta Reumatol Port 2011, 36(3):209-218.

7. Jain S, Darveau RP: Contribution of Porphyromonas gingivalis lipopolysaccharide to periodontitis. Periodontol 2000 2010, 54(1):53-70.

8. Kassem A, Henning P, Lundberg P, Souza PP, Lindholm C, Lerner UH: Porphyromonas gingivalis Stimulates Bone Resorption by Enhancing RANKL (Receptor Activator of NF-kappaB Ligand) through Activation of Toll-like Receptor 2 in Osteoblasts. J Biol Chem 2015, 290(33):20147-20158.

9. Yao W, Guan M, Jia J, Dai W, Lay YA, Amugongo S, Liu R, Olivos D, Saunders M, Lam KS et al: Reversing bone loss by directing mesenchymal stem cells to bone. Stem Cells 2013, 31(9):2003-2014.

10. Nadkarni S, McArthur S: Oestrogen and immunomodulation: new mechanisms that impact on peripheral and central immunity. Curr Opin Pharmacol 2013, 13(4):576-581.

11. Miller VM, Duckles SP: Vascular actions of estrogens: functional implications. Pharmacol Rev 2008, 60(2):210-241.

12. Ou Q, Wang X, Wang Y, Lin X: Oestrogen retains human periodontal ligament stem cells stemness in long-term culture. Cell Prolif 2018, 51(2):e12396.

13. Liu X, Kang J, Wang H, Huang T, Li C: Construction of Fluorescein Isothiocyanate-Labeled MSNs/PEG/Lycorine/Antibody as Drug Carrier for Targeting Prostate Cancer Cells. J Nanosci Nanotechnol 2018, 18(7):4471-4477.

14. Konate K, Lindberg MF, Vaissiere A, Jourdan C, Aldrian G, Margeat E, Deshayes S, Boisguerin P: Optimisation of vectorisation property: A comparative study for a secondary amphipathic peptide. Int J Pharm 2016, 
509(1-2):71-84.

15. Crombez L, Aldrian-Herrada G, Konate K, Nguyen QN, McMaster GK, Brasseur R, Heitz F, Divita G: A new potent secondary amphipathic cell-penetrating peptide for siRNA delivery into mammalian cells. Mol Ther 2009, 17(1):95-103.

16. Rittner K, Benavente A, Bompard-Sorlet A, Heitz F, Divita G, Brasseur R, Jacobs E: New basic membrane-destabilizing peptides for plasmid-based gene delivery in vitro and in vivo. Mol Ther 2002, 5(2):104-114.

17. Gugliandolo E, Fusco R, D'Amico R, Militi A, Oteri G, Wallace JL, Di Paola R, Cuzzocrea S: Anti-inflammatory effect of ATB-352, a H2S -releasing ketoprofen derivative, on lipopolysaccharide-induced periodontitis in rats. Pharmacol Res 2018, 132:220-231.

18. Manresa C, Sanz-Miralles EC, Twigg J, Bravo M: Supportive periodontal therapy (SPT) for maintaining the dentition in adults treated for periodontitis. Cochrane Database Syst Rev 2018, 1:CD009376.

19. Emampanahi M, Masoudi Rad S, Saghaeian Jazi M, Mansour Samaei N, Behnampour N, Mohammadi S, Fakhari E: Association between Interleukin-10 Gene Polymorphisms and Severe Chronic Periodontitis. Oral Dis 2019.

20. Zheng Y, Dong C, Yang J, Jin Y, Zheng W, Zhou Q, Liang Y, Bao L, Feng G, Ji J et al: Exosomal microRNA-155-5p from PDLSCs regulated Th17/Treg balance by targeting sirtuin-1 in chronic periodontitis. J Cell Physiol 2019.

21. Sun J, Dong Z, Zhang Y, He X, Fei D, Jin F, Yuan L, Li B, Jin Y: Osthole improves function of periodontitis periodontal ligament stem cells via epigenetic modification in cell sheets engineering. Sci Rep 2017, 7(1):5254.

22. Kang H, Lee MJ, Park SJ, Lee MS: Lipopolysaccharide-Preconditioned Periodontal Ligament Stem Cells Induce M1 Polarization of Macrophages through Extracellular Vesicles. Int J Mol Sci 2018, 19(12).

23. Ou $\mathrm{Q}$, Wang $\mathrm{X}$, Wang $\mathrm{Y}$, Wang $\mathrm{Y}$, Lin $\mathrm{X}$. Oestrogen retains human periodontal ligament stem cells stemness in long-ter 
m culture. Cell Prolif. 2018, 51(2):e12396.

24. Hoffmann DB, Böker KO, Schneider S, Eckermann-Felkl E,Schuder A, Komrakova M, Sehmisch S, Gruber J. In Vivo siRNA Delivery Using JC Virus-like Particles Decreases the Expression of RANKL in Rats. Mol Ther Nucleic Acids. 2016 Mar 22;5:e298.

\section{Figure legends}

Figure 1 Preparation and Characterization of MSNs, MSNs-COOH, MSNs-CADY.

Figure 2 Internalization characteristics of FITC-labeled MSNs and FITC-labeled MSNs@CADY. Blue fluorescence indicates nuclear staining with DAPI; green fluorescence indicates the localization of FITC-labeled MSNs, and red fluorescence indicates the staining of lysosomes by Lysotracker Red DND-99.

Figure 3 In Vitro Drug Releasing Property of siRNARANKL/MSNs-CADY and oestrogen/MSNs-CADY.

Figure 4 Cell Compatibility of MSNs-CADY.

Figure 5 The effect of MSNs/CADY/LRG on PDLSCs cell proliferation. 


\section{Figures}

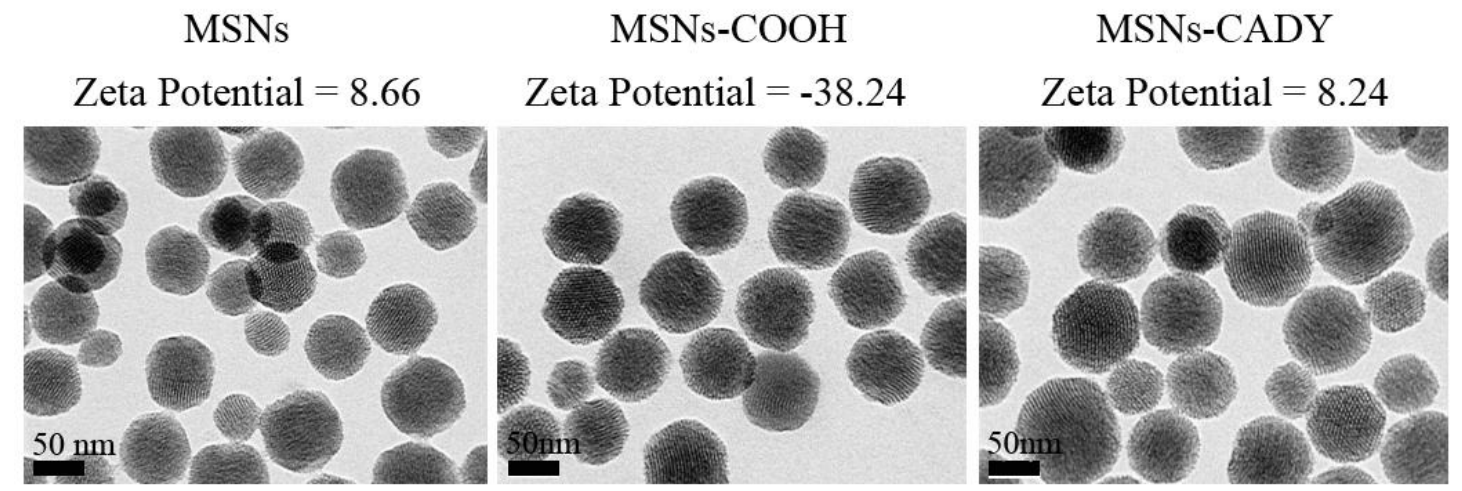

Figure 1 Preparation and Characterization of MSNs, MSNs-COOH, MSNs-CADY.

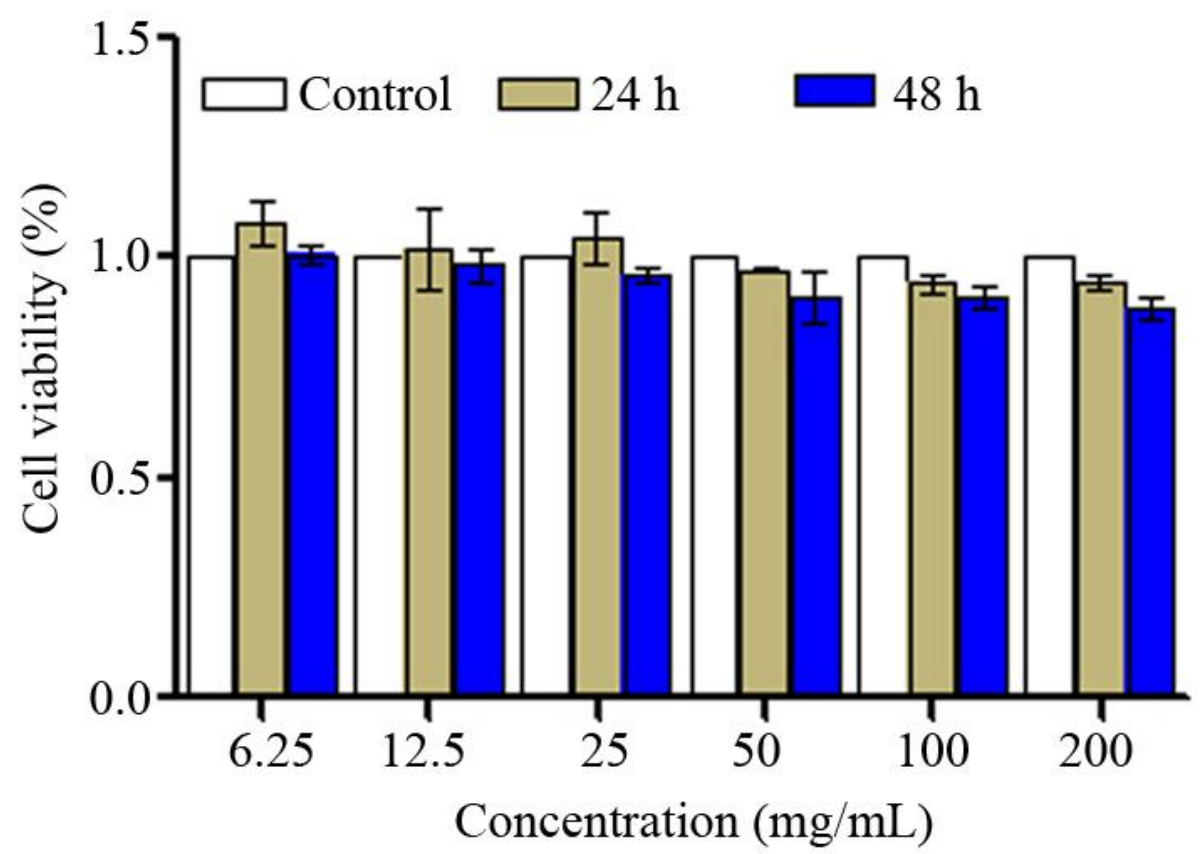

Figure 2 Internalization characteristics of FITC-labeled MSNs and FITC-labeled MSNs@CADY. Blue fluorescence indicates nuclear staining with DAPI; green fluorescence indicates the localization of FITC-labeled MSNs, and red fluorescence indicates the staining of lysosomes by Lysotracker Red DND-99. 


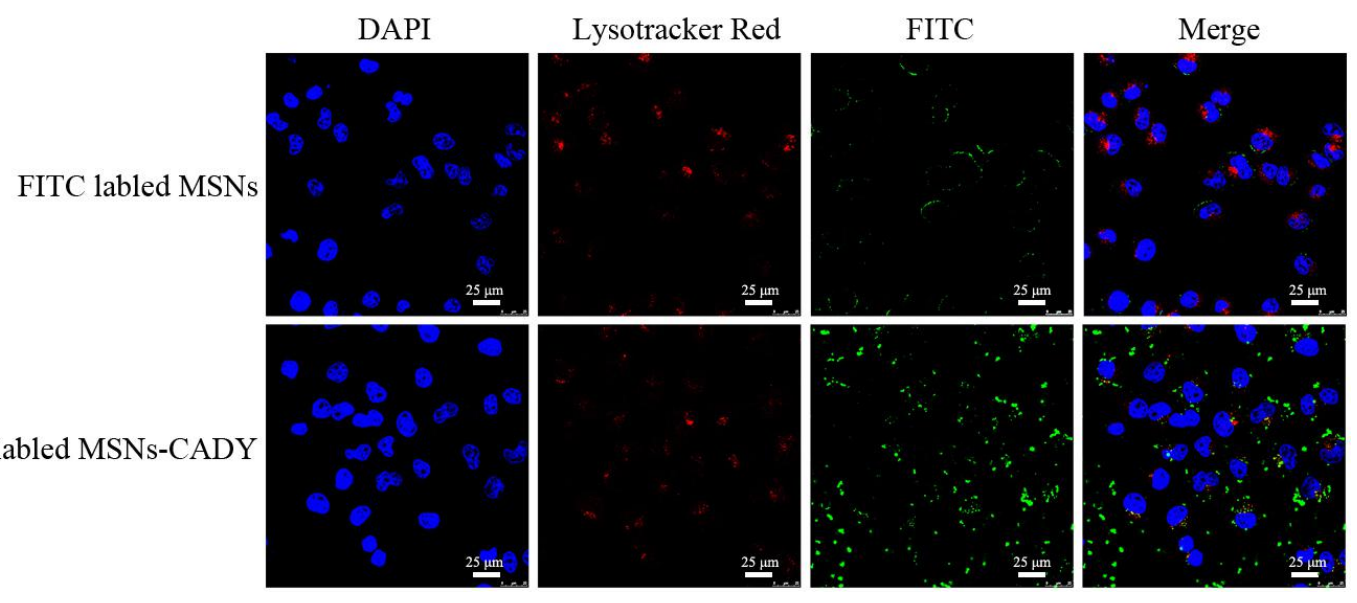

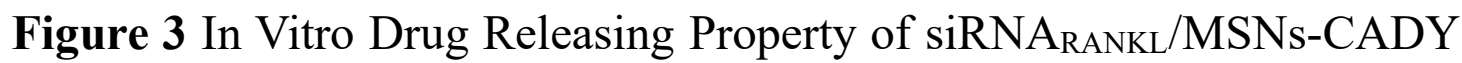
and oestrogen/MSNs-CADY.
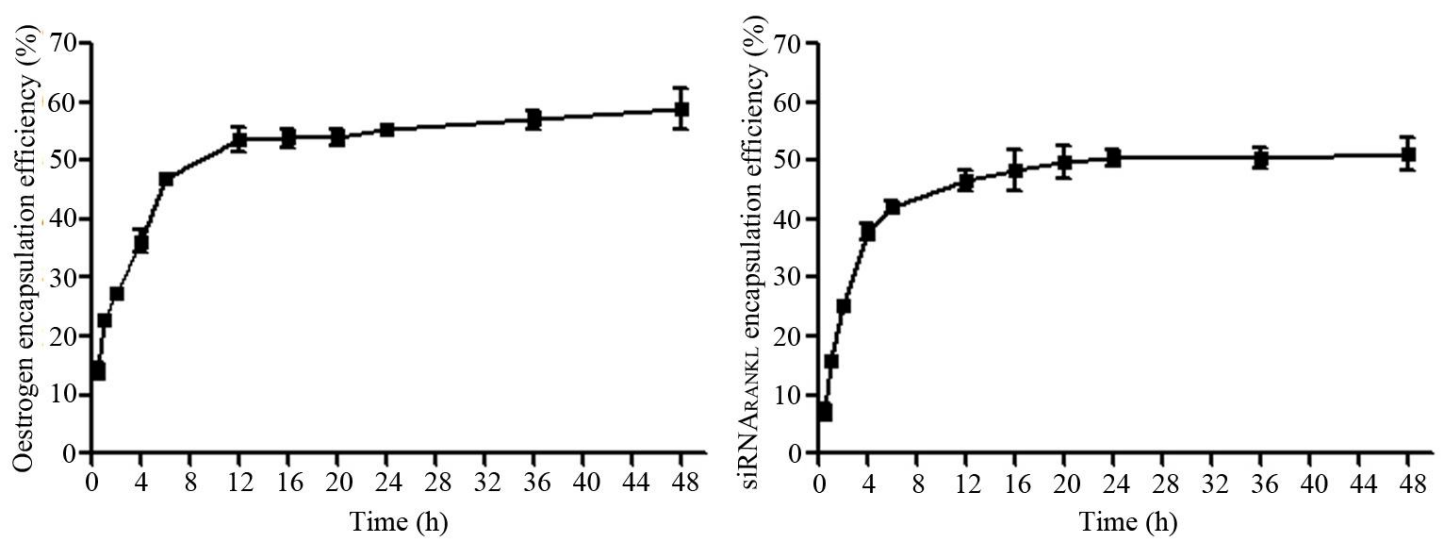

Figure 4 Cell Compatibility of MSNs-CADY.

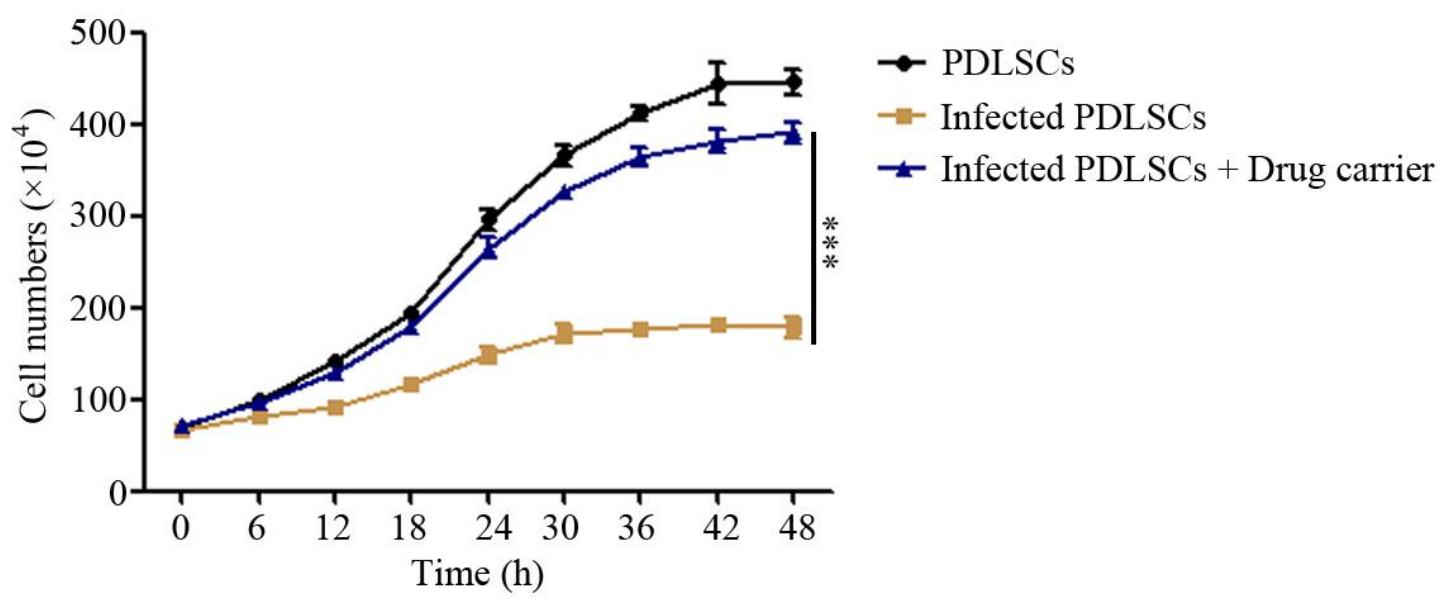

Figure 5 The effect of MSNs/CADY/LRG on PDLSCs cell proliferation. 
Figures

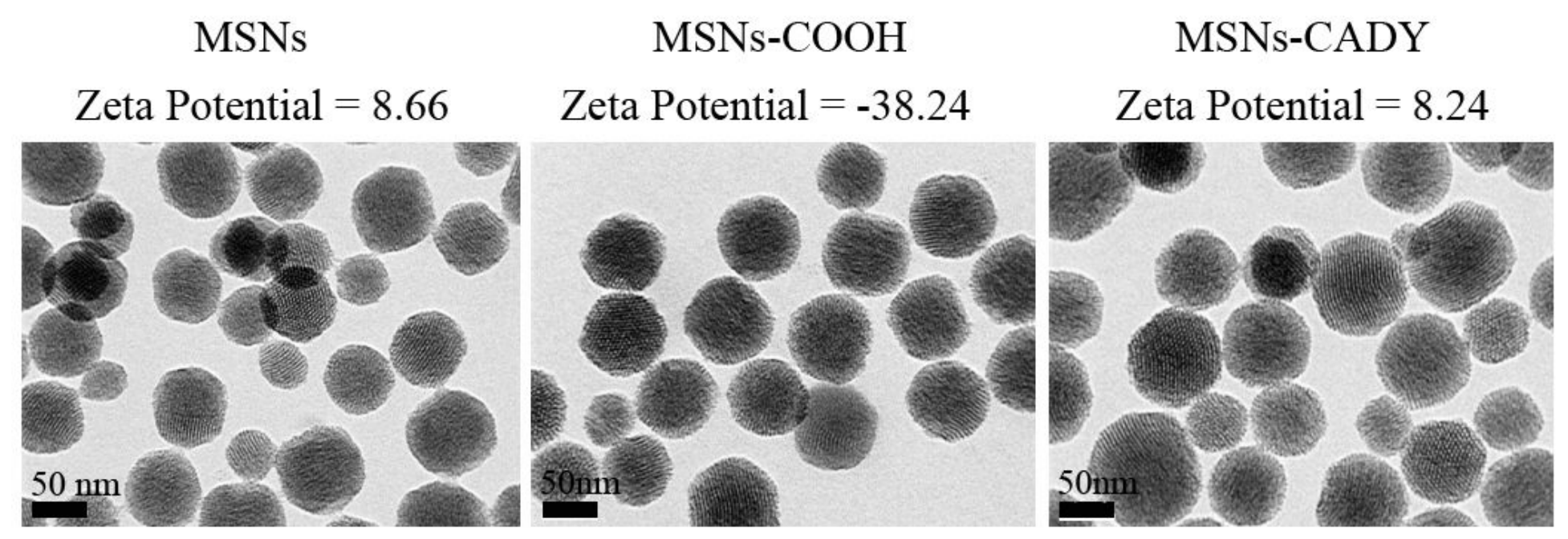

Figure 1

Preparation and Characterization of MSNs, MSNs-COOH, MSNs-CADY.

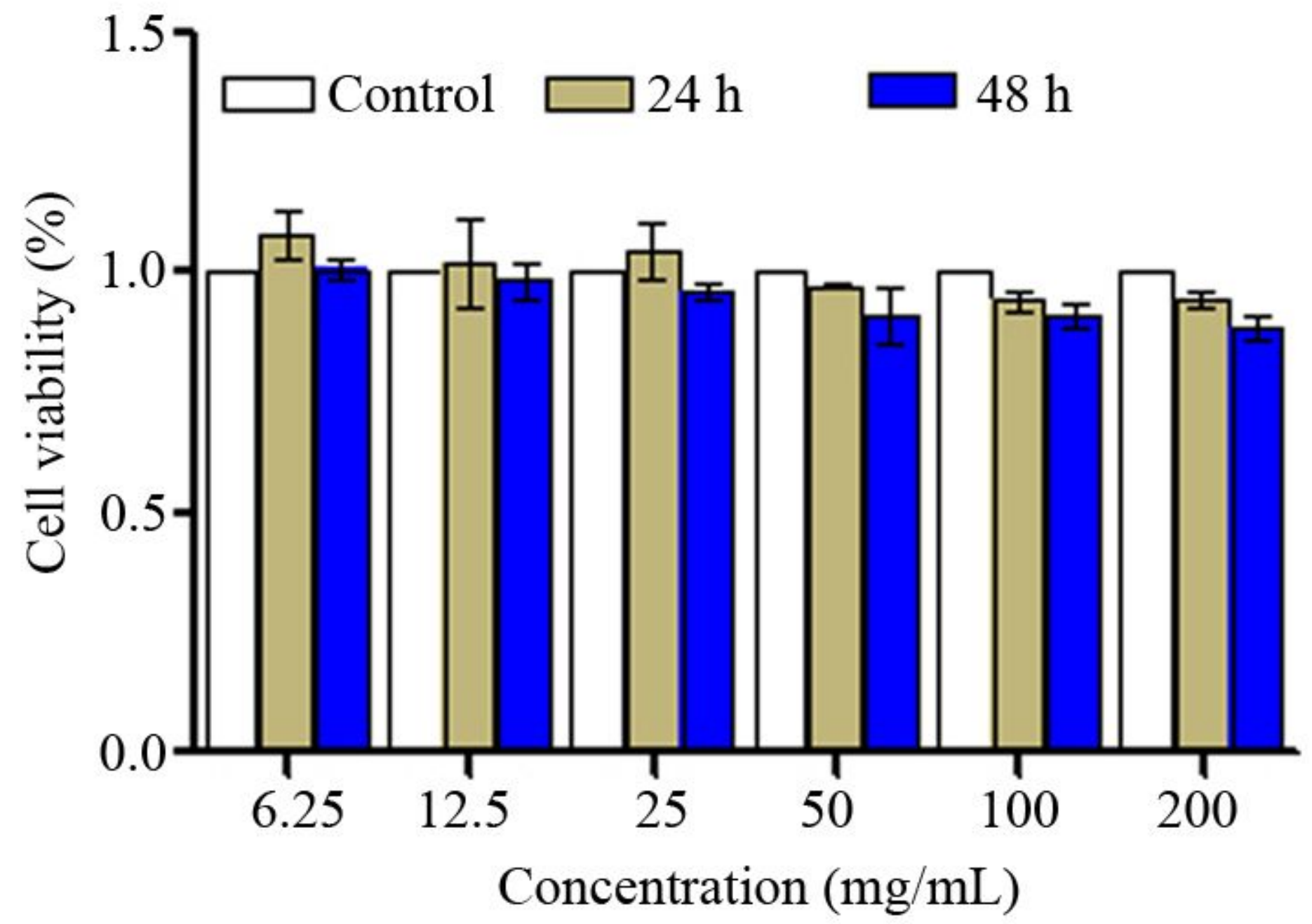

Figure 2 
Internalization characteristics of FITC-labeled MSNs and FITC-labeled MSNs@CADY. Blue fluorescence indicates nuclear staining with DAPI; green fluorescence indicates the localization of FITC-labeled MSNs, and red fluorescence indicates the staining of lysosomes by Lysotracker Red DND-99.

DAPI

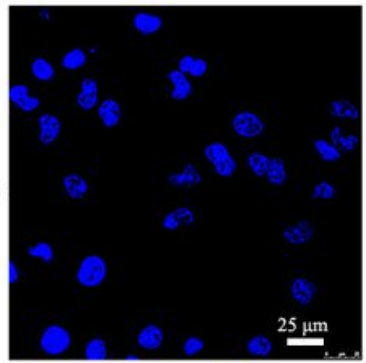

FITC labled MSNs

FITC labled MSNs-CADY

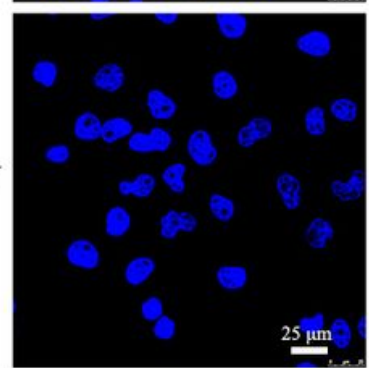

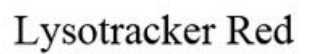
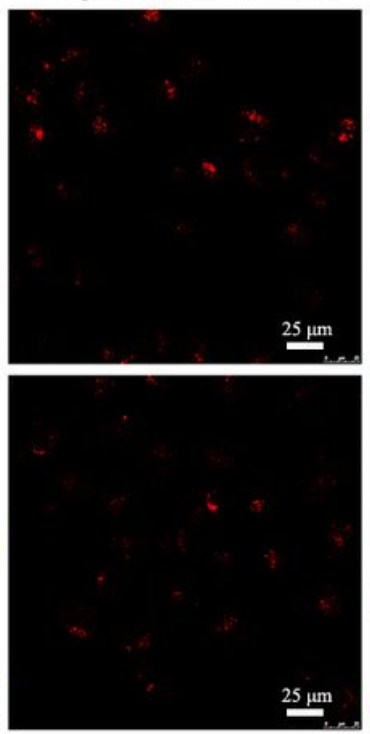

FITC
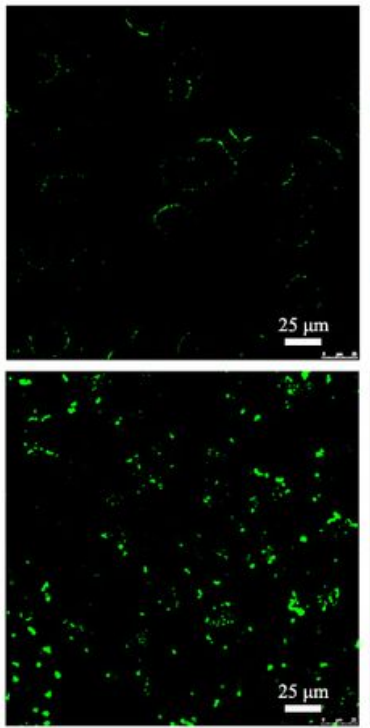

Merge

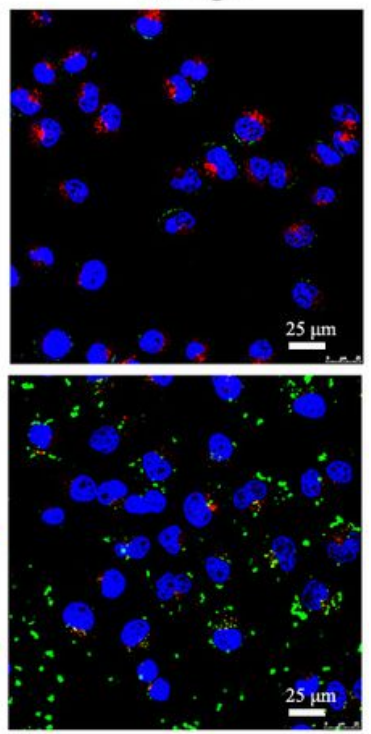

Figure 3

In Vitro Drug Releasing Property of siRNARANKL/MSNs-CADY and oestrogen/MSNs-CADY.
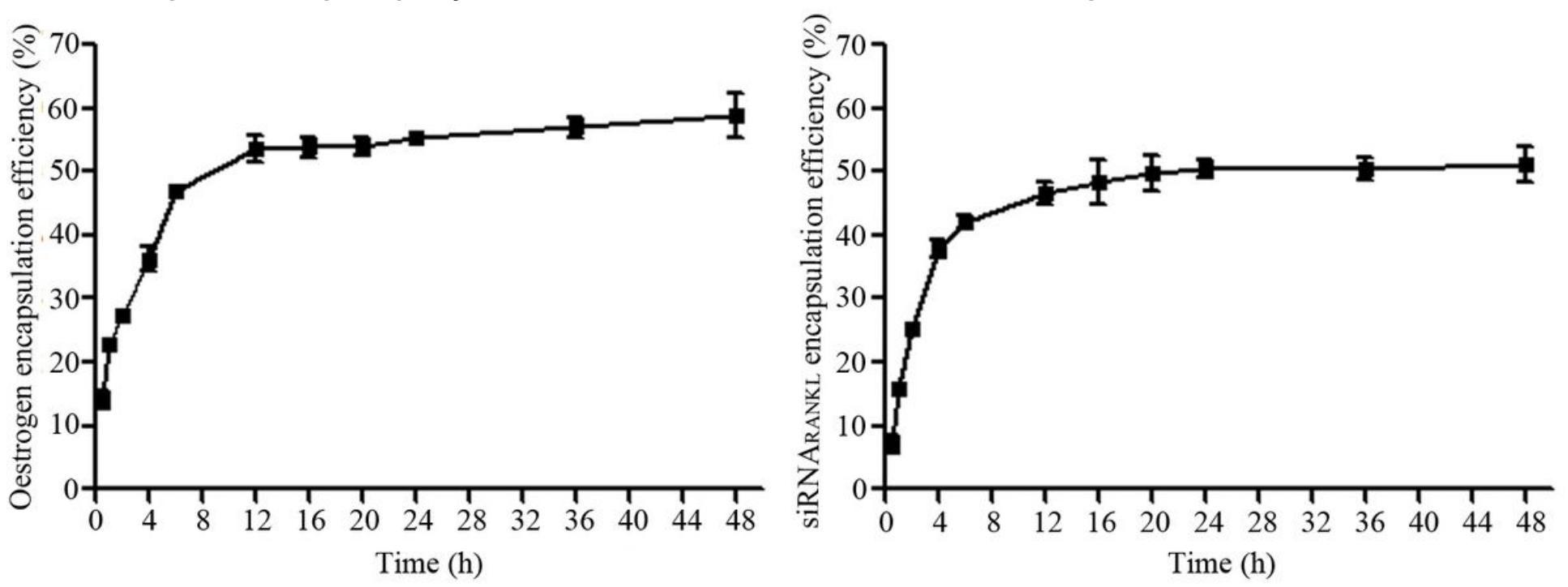

\section{Figure 4}

Cell Compatibility of MSNs-CADY. 


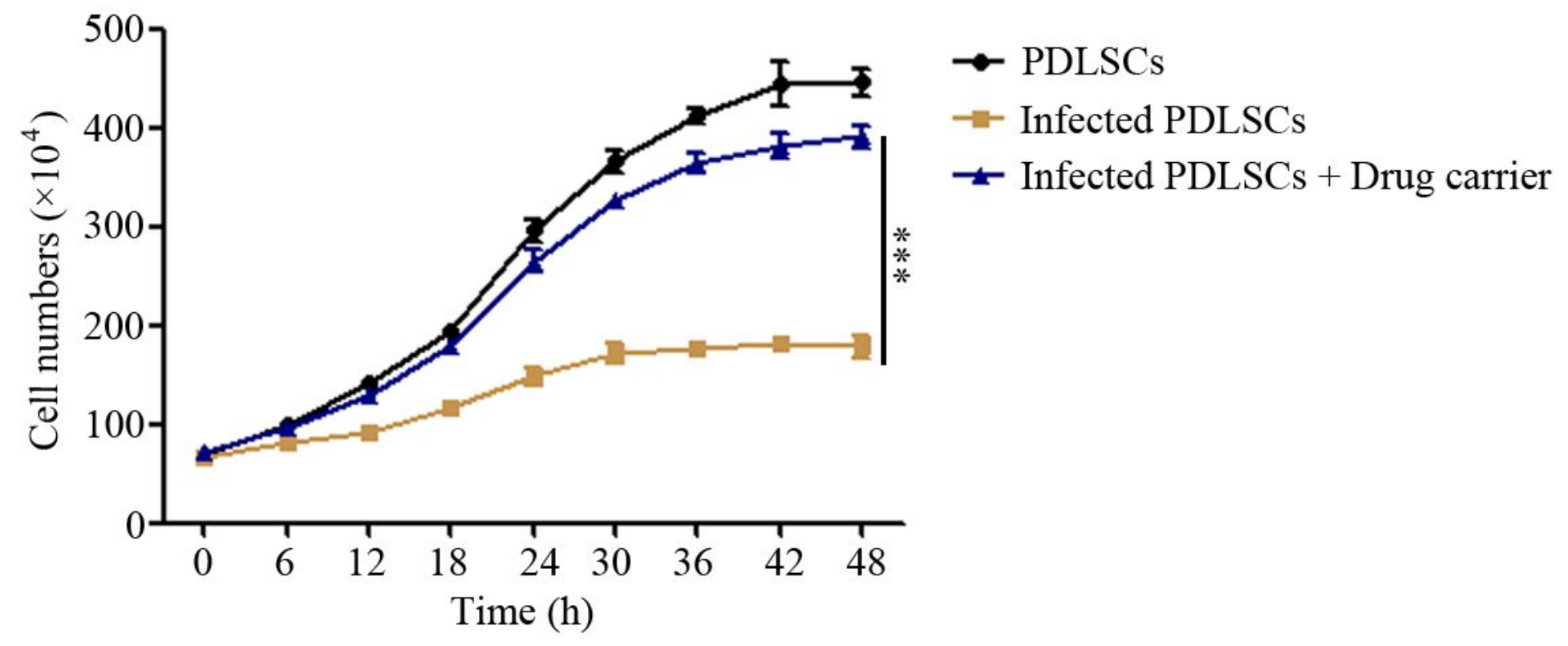

Figure 5

The effect of MSNs/CADY/LRG on PDLSCs cell proliferation. 